\title{
Cooperative Caching for Efficient Data Access in Disruption Tolerant Networks
}

\author{
Wei Gao, Member, IEEE, Guohong Cao, Fellow, IEEE, \\ Arun lyengar, Fellow, IEEE, and Mudhakar Srivatsa, Member, IEEE
}

\begin{abstract}
Disruption tolerant networks (DTNs) are characterized by low node density, unpredictable node mobility, and lack of global network information. Most of current research efforts in DTNs focus on data forwarding, but only limited work has been done on providing efficient data access to mobile users. In this paper, we propose a novel approach to support cooperative caching in DTNs, which enables the sharing and coordination of cached data among multiple nodes and reduces data access delay. Our basic idea is to intentionally cache data at a set of network central locations (NCLs), which can be easily accessed by other nodes in the network. We propose an efficient scheme that ensures appropriate NCL selection based on a probabilistic selection metric and coordinates multiple caching nodes to optimize the tradeoff between data accessibility and caching overhead. Extensive trace-driven simulations show that our approach significantly improves data access performance compared to existing schemes.
\end{abstract}

Index Terms-Cooperative caching, disruption tolerant networks, data access, network central locations, cache replacement

\section{INTRODUCTION}

$\mathrm{D}$ ISRUPTION tolerant networks (DTNs) [14] consist of mobile devices that contact each other opportunistically. Due to the low node density and unpredictable node mobility, only intermittent network connectivity exists in DTNs, and the subsequent difficulty of maintaining end-toend communication links makes it necessary to use "carryand-forward" methods for data transmission. Examples of such networks include groups of individuals moving in disaster recovery areas, military battlefields, or urban sensing applications [11]. In such networks, node mobility is exploited to let mobile nodes carry data as relays and forward data opportunistically when contacting others. The key problem is, therefore, how to determine the appropriate relay selection strategy.

Although forwarding schemes have been proposed in DTNs [4], [1], [13], there is limited research on providing efficient data access to mobile users, despite the importance of data accessibility in many mobile applications. For example, it is desirable that smartphone users can find interesting digital content from their nearby peers. In vehicular ad-hoc networks (VANETs), the availability of live traffic information will be beneficial for vehicles to avoid traffic delays.

In these applications, data are only requested by mobile users whenever needed, and requesters do not know data

-W. Gao is with the Department of Electrical Engineering and Computer Science, University of Tennessee at Knoxville, 302 Min Kao, 1520 Middle Drive, Knoxville, TN 37996. E-mail: weigao@utk.edu.

- G. Cao is with the Department of Computer Science and Engineering, Pennsylvania State University, 354G Information Science and Technology Building, University Park, PA 16802. E-mail: gcao@cse.psu.edu.

- A. Iyengar and M. Srivatsa are with the IBM T.J. Watson Research Center, 19 Skyline Dr, Hawthorne, NY 10532.

E-mail: \{aruni,msrivats\}@us.ibm.com.

Manuscript received 18 July 2011; revised 29 May 2012; accepted 18 Jan. 2013; published online 7 Mar. 2013.

For information on obtaining reprints of this article, please send e-mail to: tmc@computer.org, and reference IEEECS Log Number TMC-2011-07-0399. Digital Object Identifier no. 10.1109/TMC.2013.33. locations in advance. The destination of data is, hence, unknown when data are generated. This communication paradigm differs from publish/subscribe systems [36], [25] in which data are forwarded by broker nodes to users according to their data subscriptions. Appropriate network design is needed to ensure that data can be promptly accessed by requesters in such cases.

A common technique used to improve data access performance is caching, i.e., to cache data at appropriate network locations based on query history, so that queries in the future can be responded with less delay. Although cooperative caching has been studied for both web-based applications [15] and wireless ad hoc networks [35], [33], [16], [38] to allow sharing and coordination among multiple caching nodes, it is difficult to be realized in DTNs due to the lack of persistent network connectivity. First, the opportunistic network connectivity complicates the estimation of data transmission delay, and furthermore makes it difficult to determine appropriate caching locations for reducing data access delay. This difficulty is also raised by the incomplete information at individual nodes about query history. Second, due to the uncertainty of data transmission, multiple data copies need to be cached at different locations to ensure data accessibility. The difficulty in coordinating multiple caching nodes makes it hard to optimize the tradeoff between data accessibility and caching overhead.

In this paper, we propose a novel scheme to address the aforementioned challenges and to efficiently support cooperative caching in DTNs. Our basic idea is to intentionally cache data at a set of network central locations (NCLs), each of which corresponds to a group of mobile nodes being easily accessed by other nodes in the network. Each NCL is represented by a central node, which has high popularity in the network and is prioritized for caching data. Due to the limited caching buffer of central nodes, multiple nodes near a central node may be involved for caching, and we ensure that popular data are always cached nearer to the central 
nodes via dynamic cache replacement based on query history. Our detailed contributions are listed as follows:

- We develop an efficient approach to NCL selection in DTNs based on a probabilistic selection metric. The selected NCLs achieve high chances for prompt response to user queries with low overhead in network storage and transmission.

- We propose a data access scheme to probabilistically coordinate multiple caching nodes for responding to user queries. We furthermore optimize the tradeoff between data accessibility and caching overhead, to minimize the average number of cached data copies in the network.

- We propose a utility-based cache replacement scheme to dynamically adjust cache locations based on query history, and our scheme achieves good tradeoff between the data accessibility and access delay.

The rest of this paper is organized as follows: In Section 2, we briefly review existing work. Section 3 provides an overview of our approach and highlights our motivation of intentional caching in DTNs. Section 4 describes how to appropriately select NCLs in DTNs. Section 5 describes the details of our proposed caching scheme, and Section 6 proposes load balancing techniques among NCLs. The results of trace-driven performance evaluations are shown in Section 7, and Section 8 concludes the paper.

\section{Related Work}

Research on data forwarding in DTNs originates from Epidemic routing [34], which floods the entire network. Some later studies focus on proposing efficient relay selection metrics to approach the performance of Epidemic routing with lower forwarding cost, based on prediction of node contacts in the future. Some schemes do such prediction based on their mobility patterns, which are characterized by Kalman filter [8] or semi-Markov chains [37]. In some other schemes, node contact pattern is exploited as abstraction of node mobility pattern for better prediction accuracy [4], [24], based on the experimental [7] and theoretical [5] analysis of the node contact characteristics. The social network properties of node contact patterns, such as the centrality and community structures, have also been also exploited for relay selection in recent social-based data forwarding schemes [9], [22], [20].

The aforementioned metrics for relay selection can be applied to various forwarding strategies, which differ in the number of data copies created in the network. While the most conservative strategy [32] always keeps a single data copy and Spray-and-Wait [31] holds a fixed number of data copies, most schemes dynamically determine the number of data copies. In Compare-and-Forward [12], a relay forwards data to another node whose metric value is higher than itself. Delegation forwarding [13] reduces forwarding cost by only forwarding data to nodes with the highest metric.

Data access in DTNs, on the other hand, can be provided in various ways [28]. Data can be disseminated to appropriate users based on their interest profiles [18]. Publish/ subscribe systems [36], [25] were used for data dissemination, where social community structures are usually exploited to determine broker nodes. In other schemes [24], [2] without brokers, data items are grouped into predefined channels, and are disseminated based on users' subscriptions to these channels.

Caching is another way to provide data access. Cooperative caching in wireless ad hoc networks was studied in [35], in which each node caches pass-by data based on data popularity, so that queries in the future can be responded with less delay. Caching locations are selected incidentally among all the network nodes. Some research efforts [27], [21] have been made for caching in DTNs, but they only improve data accessibility from infrastructure network such as WiFi access points (APs) [21] or Internet [27]. Peer-to-peer data sharing and access among mobile users are generally neglected.

Distributed determination of caching policies for minimizing data access delay has been studied in DTNs [29], [23], assuming simplified network conditions. In [29], it is assumed that all the nodes contact each other with the same rate. In [23], users are artificially partitioned into several classes such that users in the same class are identical. In [19], data are intentionally cached at appropriate network locations with generic data and query models, but these caching locations are determined based on global network knowledge. Comparatively, in this paper, we propose to support cooperative caching in a fully distributed manner in DTNs, with heterogeneous node contact patterns and behaviors.

\section{Overview}

\subsection{Motivation}

A requester queries the network for data access, and the data source or caching nodes reply to the requester with data after having received the query. The key difference between caching strategies in wireless ad hoc networks and DTNs is illustrated in Fig. 1. Note that each node has limited space for caching. Otherwise, data can be cached everywhere, and it is trivial to design different caching strategies.

The design of caching strategy in wireless ad hoc networks benefits from the assumption of existing end-toend paths among mobile nodes, and the path from a requester to the data source remains unchanged during data access in most cases. Such assumption enables any intermediate node on the path to cache the pass-by data. For example, in Fig. 1a, $C$ forwards all the three queries to data sources $A$ and $B$, and also forwards data $d_{1}$ and $d_{2}$ to the requesters. In case of limited cache space, $C$ caches the more popular data $d_{1}$ based on query history, and similarly data $d_{2}$ are cached at node $K$. In general, any node could cache the pass-by data incidentally.

However, the effectiveness of such an incidental caching strategy is seriously impaired in DTNs, which do not assume any persistent network connectivity. Since data are forwarded via opportunistic contacts, the query and replied data may take different routes, and it is difficult for nodes to collect the information about query history and make caching decision. For example, in Fig. 1b, after having forwarded query $q_{2}$ to $A$, node $C$ loses its connection to $G$, and cannot cache data $d_{1}$ replied to 


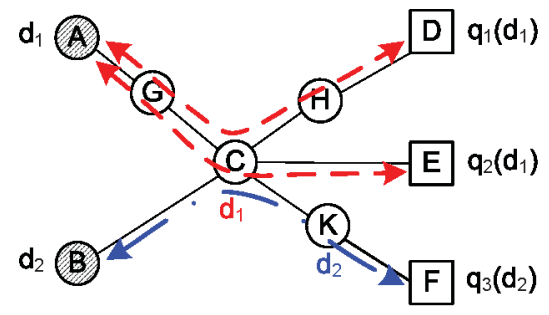

(a) Wireless ad-hoc networks

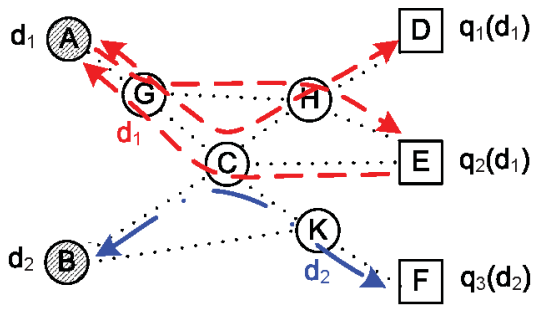

(b) Disruption-Tolerant Networks

Fig. 1. Caching strategies in different network environments. Data $d_{1}$ generated by node $A$ are requested by nodes $D$ and $E$, and $d_{2}$ generated by node $B$ are requested by node $F$. A solid line in (a) between nodes indicates a wireless link, and a dotted line in (b) indicates that two nodes opportunistically contact each other.

requester $E$. Node $H$ which forwards the replied data to $E$ does not cache the pass-by data $d_{1}$ either because it did not record query $q_{2}$ and considers $d_{1}$ less popular. In this case, $d_{1}$ will be cached at node $G$, and hence needs longer time to be replied to the requester.

Our basic solution to improve caching performance in DTNs is to restrain the scope of nodes being involved for caching. Instead of being incidentally cached "anywhere," data are intentionally cached only at specific nodes. These nodes are carefully selected to ensure data accessibility, and constraining the scope of caching locations reduces the complexity of maintaining query history and making caching decision.

\subsection{Network Model}

Opportunistic contacts in DTNs are described by a network contact graph $G(V, E)$, where the stochastic contact process between a node pair $i, j \in V$ is modeled as an edge $e_{i j} \in E$. We assume that node contacts are symmetric; i.e., node $j$ contacts $i$ whenever $i$ contacts $j$, and the network contact graph is, therefore, undirected. The characteristics of an edge $e_{i j} \in E$ are determined by the properties of intercontact time among nodes. Similar to previous work [1], [39], we consider the pairwise node intercontact time as exponentially distributed. Contacts between nodes $i$ and $j$ then form a Poisson process with contact rate $\lambda_{i j}$, which is calculated in real time from the cumulative contacts between nodes $i$ and $j$ because the network starts. In the rest of this paper, we call the node set $\left\{j \mid \lambda_{i j}>0\right\} \subseteq V$ as the contacted neighbors of $i$.

\subsection{The Big Picture}

We consider a general caching scenario, in which each node may generate data with a globally unique identifier ${ }^{1}$ and finite lifetime, and may also request other data by sending queries with a finite time constraint. In practice, such scenario could correspond to various types of disaster environments, which contain mobile users being rescued after hurricane, fire accidents, or earthquake. In these scenarios, the cellular 3G infrastructure may usually be unavailable, or provide too limited bandwidth to transmit data traffic. Instead, mobile users rely on their opportunistic contacts for accessing data, which can be live weather report,

1. We assign the identifier of each data item as combination of data source's ID and an nondecreasing sequence number, which is maintained by data source and increases whenever a new data item is generated by the source. traffic condition, or government rescue plan. Similarly, a platoon of soldiers in the military battlefield may lose the satellite connection due to enemy attacks, and have to distribute battle reports via their opportunistic contacts.

In these scenarios, either a data item or a query is described by a set of keywords over a keyword space [18], so that caching nodes can determine the appropriate data that a user is interested in. In these scenarios, data requesters are randomly distributed in the network. We focus on efficiently utilizing the available node buffer to optimize the overall caching performance, which is measured by the successful ratio and delay for mobile users to access different data items.

Our basic idea is to intentionally cache data only at a specific set of NCLs, which can be easily accessed by other nodes in the network. Queries are forwarded to NCLs for data access. ${ }^{2}$ The big picture of our proposed scheme is illustrated in Fig. 2. Each NCL is represented by a central node, which corresponds to a star in Fig. 2. The push and pull caching strategies conjoin at the NCLs. The data source $S$ actively pushes its generated data toward the NCLs, and the central nodes $C_{1}$ and $C_{2}$ of NCLs are prioritized for caching data. If the buffer of a central node $C_{1}$ is full, data are cached at another node $A$ near $C_{1}$. Multiple nodes at a NCL may be involved for caching, and a NCL, hence, corresponds to a connected subgraph of the network contact graph $G$, as the dashed circles illustrated in Fig. 2. Note that NCLs may be overlapping with each other, and a node being involved for caching may belong to multiple NCLs simultaneously. A requester $R$ pulls data by querying NCLs, and data copies from multiple NCLs are returned to ensure prompt data access. Particularly, some NCL such as $C_{2}$ may be too far from $R$ to receive the query on time, and does not respond with data. In this case, data accessibility is determined by both node contact frequency and data lifetime.

Nodes in DTNs are well motivated to contribute their local resources for caching data because the cached data provide prompt data access to the caching nodes themselves. As illustrated by Fig. 2, since the central nodes representing NCLs are prioritized for caching data, the closer a requester is to a central node, the sooner its queries are responded by the corresponding NCL. The delay for responding to queries generated from central nodes is, obviously, the shortest.

2. Note that our scheme is different from publish/subscribe system, in which published data are forwarded to subscribers instead of being cached by brokers. 


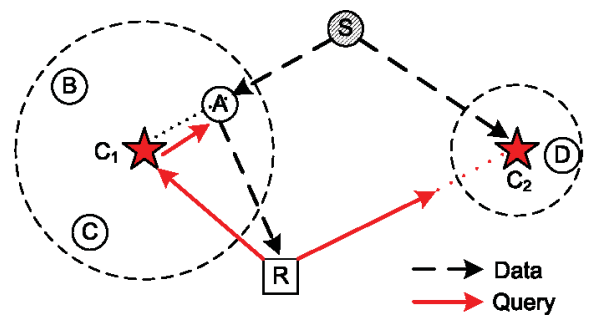

Fig. 2. The big picture of intentional caching.

\section{Network Central locations}

In this section, we describe how to select NCLs based on a probabilistic metric evaluating the data transmission delay among nodes in DTNs; we validate the applicability of such metric in practice based on the heterogeneity of node contact pattern in realistic DTN traces. Furthermore, we propose detailed methods for selecting NCLs in practice based on different availability of network information.

\subsection{NCL Selection Metric}

We first define the multihop opportunistic connection on network contact graph $G=(V, E)$.

Definition 1 (Opportunistic path). A r-hop opportunistic path $P_{A B}=\left(V_{P}, E_{P}\right)$ between nodes $A$ and $B$ consists of a node set $V_{P}=\left\{A, N_{1}, N_{2}, \ldots, N_{r-1}, B\right\} \subset V$ and an edge set $E_{P}=\left\{e_{1}, e_{2}, \ldots, e_{r}\right\} \subset E$ with edge weights $\left\{\lambda_{1}, \lambda_{2}, . ., \lambda_{r}\right\}$. Path weight $p_{A B}(T)$ is the probability that data are opportunistically transmitted from $A$ to $B$ along $P_{A B}$ within time $T$.

An opportunistic path is illustrated in Fig. 3. As described in Section 3.2, the intercontact time $X_{k}$ between nodes $N_{k}$ and $N_{k+1}$ on $P_{A B}$ follows exponential distribution with probability density function (PDF) $p_{X_{k}}(x)=\lambda_{k} e^{-\lambda_{k} x}$. Hence, the time needed to transmit data from $A$ to $B$ is $Y=\sum_{k=1}^{r} X_{k}$ following a hypoexponential distribution [30], such that

$$
p_{Y}(x)=\sum_{k=1}^{r} C_{k}^{(r)} p_{X_{k}}(x),
$$

where the coefficients $C_{k}^{(r)}=\prod_{s=1, s \neq k}^{r} \frac{\lambda_{s}}{\lambda_{s}-\lambda_{k}}$.

From (1), the path weight is written as

$$
p_{A B}(T)=\int_{0}^{T} p_{Y}(x) d x=\sum_{k=1}^{r} C_{k}^{(r)} \cdot\left(1-e^{-\lambda_{k} T}\right),
$$

and the data transmission delay between two nodes $A$ and $B$, indicated by the random variable $Y$, is measured by the weight of the shortest opportunistic path between the two nodes. In practice, mobile nodes maintain the information about shortest opportunistic paths between each other in a distance-vector manner when they come into contact.

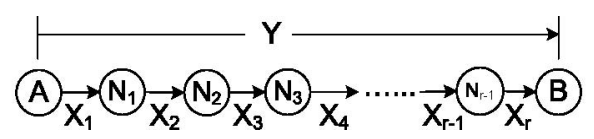

Fig. 3. Opportunistic path.
TABLE 1

Trace Summary

\begin{tabular}{c|cccc}
\hline Trace & Infocom05 & Infocom06 & MIT Reality & UCSD \\
\hline \hline Network type & Bluetooth & Bluetooth & Bluetooth & WiFi \\
No. devices & 41 & 78 & 97 & 275 \\
No. contacts & 22,459 & 182,951 & 114,046 & 123,225 \\
Duration (days) & 3 & 4 & 246 & 77 \\
Granularity (secs) & 120 & 120 & 300 & 20 \\
Avg. inter-contact & 3.43 & 1.83 & 84.13 & 47.17 \\
time (hours) & & & & \\
\hline
\end{tabular}

The metric $C_{i}$ for a node $i$ to be selected as a central node to represent a NCL is then defined as follows:

$$
C_{i}=\frac{1}{|V|} \cdot \sum_{j \in V} p_{i j}(T)
$$

where we define that $p_{i i}(T)=0$. This metric indicates the average probability that data can be transmitted from a random node to node $i$ within time $T$. From (3), it is obvious that the value of $C_{i}$ decreases exponentially when $T$ decreases.

\subsection{Trace-Based Validation}

The practical applicability of the aforementioned NCL selection metric is based on the heterogeneity of node contact patterns, such that nodes in DTNs differ in their popularity and few nodes contact many others frequently. In this section, we validate this applicability using realistic DTN traces.

These traces record contacts among users carrying mobile devices in conference sites and university campuses. The mobile devices, including Mica2 sensors or smartphones, are distributed to users being participated into the experiment. Devices equipped with Bluetooth interface periodically detect their peers nearby, and a contact is recorded when two devices move close to each other. Devices equipped with WiFi interface search for nearby WiFi APs and associate themselves to APs with the best signal strength. A contact is recorded when two devices are associated with the same AP. The detected contacts are recorded in the local storage of mobile devices. After the experiment ends, these devices are called back so that the recorded contacts are processed and analyzed. The traces are summarized in Table 1.

We calculate pairwise node contact rates based on their cumulative contacts during the entire trace. According to (2), inappropriate values of $T$ make $C_{i}$ close to 0 or 1 . Instead, values of $T$ are adaptively determined in different traces to ensure the differentiation of NCL selection metric values of nodes. $T$ is set as 1 hour for the two Infocom traces, one week for the MIT Reality trace, and three days for the UCSD trace.

The results in Fig. 4 show that the distributions of NCL selection metric values are skewed in all traces, and the metric values of few nodes are much higher than that of others. This difference can be up to $10 \mathrm{fold}$, and suggests that our proposed NCL selection metric efficiently indicates the heterogeneity of node contact pattern. Hence, this metric ensures the selected NCLs can be easily accessed by other nodes. 


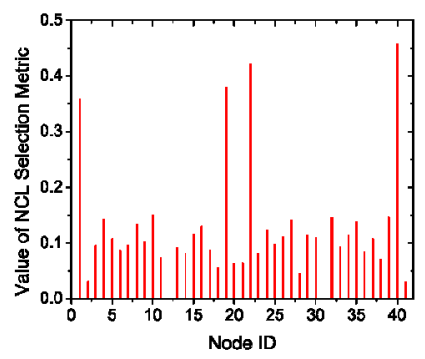

(a) Infocom05

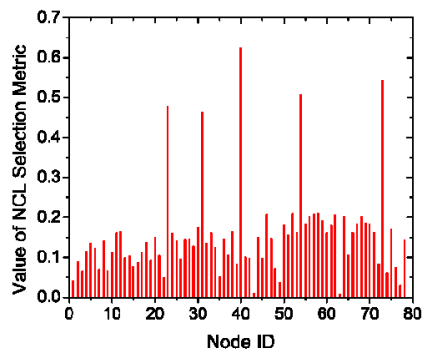

(b) Infocom06

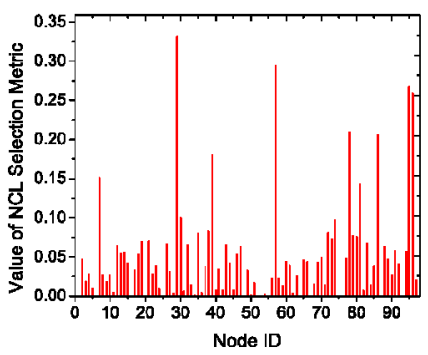

(c) MIT Reality

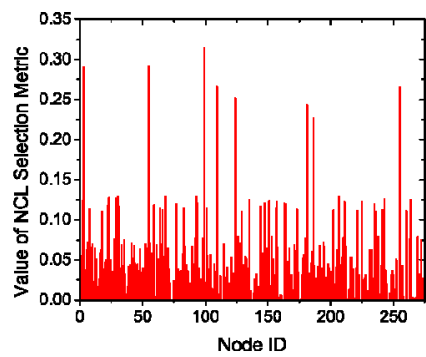

(d) UCSD

Fig. 4. Values of NCL selection metric on realistic DTN traces.

\subsection{Practical NCL Selection}

In this section, we propose methods for selecting the required $K$ NCLs in practice based on the NCL selection metric proposed in Section 4.1. We consider $K$ as a predefined parameter determined by the network performance requirements, which will be discussed later in Section 5.5 in more detail.

In general, network information about the pairwise node contact rates and shortest opportunistic paths among mobile nodes are required to calculate the metric values of mobile nodes according to (3). However, the maintenance of such network information is expensive in DTNs due to the lack of persistent end-to-end network connectivity. As a result, we will first focus on selecting NCLs with the assumption of complete network information from the global perspective. Afterwards, we propose distributed NCL selection methods that efficiently approximate global selection results and can operate on individual nodes in an autonomous manner.

\subsubsection{Global Selection}

When global network knowledge about the pairwise node contact rates and shortest opportunistic paths among mobile nodes are available, central nodes representing NCLs can be selected sequentially by the network administrator before data access. Let $\mathbb{N}_{C}$ denote the set of selected central nodes; every time the node in $V \backslash \mathbb{N}_{C}$ with the highest metric value is selected as the next central node, until the required $K$ central nodes are selected. In particular, we exclude the set $\mathbb{N}_{C}$ of existing central nodes from calculating $C_{i}$ in (3), i.e.,

$$
C_{i}=\frac{1}{\left|V \backslash \mathbb{N}_{C}\right|} \cdot \sum_{j \in V \backslash \mathbb{N}_{C}} p_{i j}(T) .
$$

By doing so, we ensure that the selected central nodes will not be clustered on the network contact graph. The parameter $T$ used in (4) is determined by the average node contact frequency. This parameter is generally trace dependent and was discussed in Section 4.2 for different DTN traces.

A network warm-up period is reserved for nodes to collect information and calculate their pairwise contact rates as described in Section 3.2, and central nodes are selected after the warm-up period ends. Data are unable to be intentionally cached at the NCLs during the warm-up period. Instead, data are incidentally cached by nodes in the

network, as described in Section 3.1, for data access. In particular, every requester sends queries directly to the data source, and caches the received data locally for responding other pass-by queries in the future.

After the central nodes representing NCLs are selected, the network administrator is responsible for notifying each node in the network about the information of NCLs via cellular or satellite links. Since each node is only notified about the identifiers of central nodes, this notification is costeffective without producing noticeable communication overhead, even in cases where the central nodes frequently change. Note that the central nodes are selected due to their popularity in the network, rather than their computation or storage capabilities. Therefore, in general, we assume that the central nodes have similar capabilities in computation, data transmission, and storage with other nodes in DTNs. Later in Section 6, we will furthermore study load balancing among central nodes when their local resources are depleted.

\subsubsection{Distributed Selection}

When global network knowledge is unavailable, a node maintains information about pairwise contact rates and shortest opportunistic paths to other nodes via opportunistic contacts. According to Definition 1 of the opportunistic path, Lemma 1 formally shows that the distributed maintenance of opportunistic paths in DTNs cannot be done in an iterative manner.

Lemma 1. There does not exist a function $f(\lambda, T)$, such that for any opportunistic path $P_{A B}=\left(A, N_{1}, \ldots, N_{r-1}, B\right)$ with edge weights $\left\{\lambda_{1}, \lambda_{2}, \ldots, \lambda_{r}\right\}$,

$$
p_{A B}(T)=p_{A N_{r-1}}(T) \otimes f\left(\lambda_{r}, T\right),
$$

where $\otimes$ can be any arbitrary arithmetic operation.

Proof. The difficulty of calculating $p_{A B}(T)$ in an iterative manner mainly comes from the properties of the coefficients $C_{k}^{(r)}$ in (2). When a new edge $\left(N_{r-1}, B\right)$ with weight $\lambda_{r}$ is added into a path $A N_{r-1}$, such coefficients are modified as

$$
C_{k}^{(r)}= \begin{cases}C_{k}^{(r-1)} \cdot \frac{\lambda_{k}}{\lambda_{r}-\lambda_{k}}, & k \neq r, \\ \prod_{s=1}^{r-1} \frac{\lambda_{s}}{\lambda_{s}-\lambda_{r}}, & k=r .\end{cases}
$$

We have two observations from (5). First, each coefficient $C_{k}^{(r)}(k \neq r)$ is updated by multiplying a distinct value $\frac{\lambda_{k}}{\lambda_{r+1}-\lambda_{k}}$. Second, calculation of $C_{r}^{(r)}$ 
involves all the edge weights $\lambda_{1}, \ldots, \lambda_{r-1}$. Both of them make it impossible to calculate $p_{A B}(T)$ solely from $p_{A N_{r-1}}(T)$ and $\lambda_{r}$

Instead, a node $i$ needs to maintain the complete opportunistic paths to other nodes in the network. Initially, each node only has the information about its contacted neighbors. When a node $A$ contacts another node $B$, they exchange and update their opportunistic path tables. More specifically, for a record of node $C$ in $B^{\prime}$ s table, if $C$ has not been recorded at $A, A$ adds this record into its own table. Otherwise, if the path to $C$ recorded by $B$ has larger weight than that recorded by $A, A$ updates its local record about $C$.

Being similar with global NCL selection, a network warm-up period is reserved for nodes to exchange and maintain necessary information about opportunistic paths to others. However, a longer warm-up period is needed for distributed NCL selection because multihop opportunistic data transmission is required for distributed maintenance of such information.

Afterwards, each node in the network autonomously calculates the value of its NCL selection metric according to (3) and broadcasts this value to the network. After a predefined broadcasting period, a node having received these values then selects the nodes with the $K$ highest metric values as the central nodes representing NCLs.

However, due to the uncertainty of opportunistic data transmission in DTNs, the broadcasting range of a particular node may not cover the entire network. Such broadcasting range in DTNs can be formally bounded by the following lemma.

Lemma 2. Suppose that node A broadcast its metric value at time $t_{0}$ and $\mathbb{S}_{A}\left(t_{0}, t\right)$ denotes the set of nodes which have received $A$ 's information by time $t_{0}+t$. We have

$$
\mathbb{P}\left(\left|\mathbf{S}_{A}\left(t_{0}, t\right)\right| \geq n\right) \geq\left(1-e^{-h_{G} t}\right)^{n-1},
$$

where

$$
h_{G}=\min _{U \subset V} \frac{\sum_{i \in U, j \in V \backslash U} \lambda_{i j}}{\min (|U|,|V \backslash U|)}
$$

is an invariant only depending on the characteristics of the network contact graph.

Proof. Letting $T_{k}$ be the time by which at least $k$ nodes in the network have received the information of node $A$, i.e., $T_{k}=\inf \left\{t\right.$, s.t. $\left.\left|\mathbf{S}_{A}\left(t_{0}, t\right)\right| \geq k\right\}$, we can easily have

$$
\mathbb{P}\left(T_{k}-T_{k-1} \leq t\right)=1-e^{-\Lambda t},
$$

which means that the random variable $T_{k}-T_{k-1}$ is exponentially distributed with parameter $\Lambda=\sum_{i \in S, j \in V \backslash S} \lambda_{i j}$, and $S=\mathbb{S}_{A}\left(t_{0}, T_{k-1}\right)$. According to definition of $h_{G}$ in (7),

$$
\Lambda \geq h_{G} \cdot \min (|S|,|V \backslash S|) \geq(k-1) h_{G},
$$

and, hence, we have

$$
\mathbb{P}\left(\left|\mathbb{S}_{A}\left(t_{0}, t\right)\right| \geq k\right) \geq 1-\mathbb{P}\left(\sum_{j=1}^{k-1} X_{j} \geq t\right),
$$

where $X_{j}$ is exponentially distributed with parameter $j h_{G}$. This lemma is then proved via induction over $k$ based on (8).

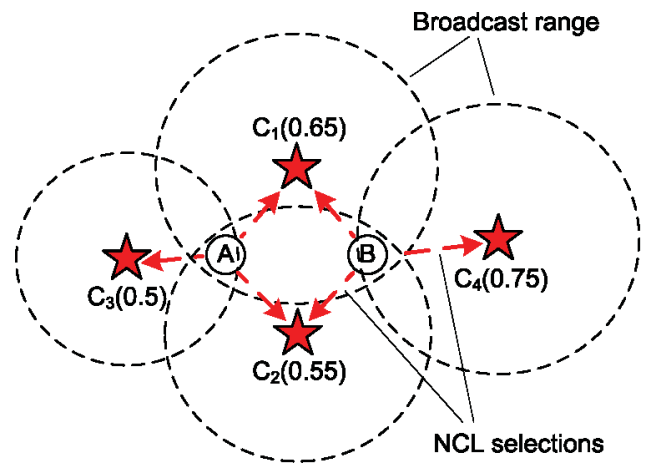

Fig. 5. Inconsistency in distributed NCL selection when $K=3$.

From Lemma 2, we can see that the metric value of a particular node can only be broadcasted to the entire network after a sufficiently long period of time. In this case, the distributed NCL selections made at individual nodes may be inconsistent. Such inconsistency is illustrated in Fig. 5, where the numbers in brackets indicate the values of nodes' NCL selection metric. Due to the limited time for broadcasting, node $A$ is unaware of $C_{4}$ which has a high metric value of 0.75 , and hence selects $C_{3}$ with a lower metric value as the central node when $K=3$. This suboptimal selection $A$ made then becomes inconsistent with $B$ 's selections.

Based on Lemma 2, we have the following theorem which evaluates the occurrence probability of such inconsistency.

Theorem 1. After a broadcasting period $T$, the probability that the NCL selections made by two arbitrary nodes $A$ and $B$ in the network are inconsistent is no larger than $1-(1-$ $2 p(1-p))^{K}$, where $K$ is the predefined number of NCLs, and

$$
p=\sum_{n=1}^{N}\left(1-e^{-h_{G} T}\right)^{n-1} \cdot\left(1-\frac{n}{N}\right) .
$$

Proof. Suppose that $N_{1}, \ldots, N_{K}$ are the central nodes being selected with global network information. For each $N_{i}$, if the information of its metric value has been received by $n$ nodes after the broadcasting period $T$, node $A$ has the probability $1-n / N$ for not having received such information. Therefore, $p$ in (9) indicates the probability that the information of $N_{i}$ has not been received by node $A$, according to Lemma 2.

It is easy to see that the same probability $p$ also applies to node $B$. As a result, $1-2 p(1-p)$ provides an upper bound on the probability that the NCL selections made by nodes $A$ and $B$ are inconsistent on any $N_{i}$ for $1 \leq i \leq K$, and this theorem is therefore proved.

The inconsistency illustrated in Fig. 5 can generally be solved by two methods. The first and more straightforward method is to extend the broadcasting period, so that each node is aware of the metric values of all the other nodes in the network. However, this method may be impractical in some mobile applications with strict requirements of timeliness. Another alternative is to opportunistically correct suboptimal NCL selections when nodes contact each other. 


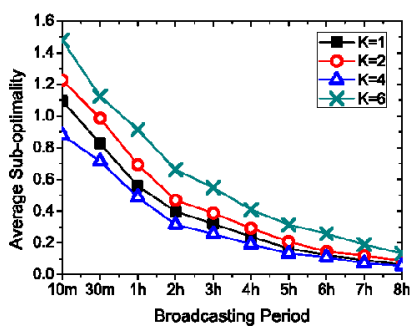

(a) Infocom 05

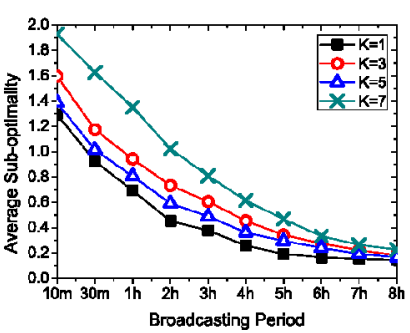

(b) Infocom06

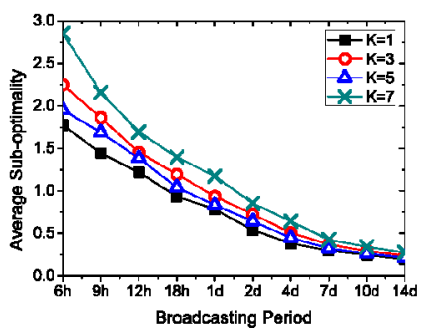

(c) MIT Reality

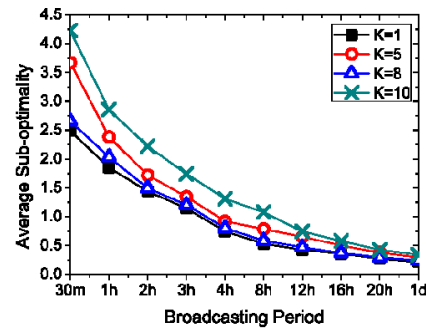

(d) UCSD

Fig. 6. Suboptimality of distributed NCL selection on realistic DTN traces.

More specifically, each node maintains the list of its selected central nodes and their metric values. They exchange such information whenever they contact other and replace the selected central nodes if better ones are found. For example in Fig. 5, node $A$ is able to find out that $C_{4}$ is a better choice as a central node when it contacts node $B$.

At last, we evaluate the performance of distributed NCL selection using realistic DTN traces. Due to the aforementioned inconsistency, central nodes selected by individual nodes may be suboptimal, and we evaluate this suboptimality at a node $i$ as $\frac{1}{K} \sum_{j=1}^{K}\left|I_{j}-j\right|$, where the $j$ th central node selected by node $i$ has the $I_{j}$ th largest metric value in the network. ${ }^{3}$ The average suboptimality over all the nodes in the network with different broadcasting periods $T$ is shown in Fig. 6. In general, Fig. 6 shows that the performance of distributed NCL selection is closely related with the length of $T$. The selected central nodes are far from optimal when $T$ is small, but will be quickly improved when $T$ increases. When $T$ is sufficiently large, the performance of distributed NCL selection closely approximates that of global selection.

Fig. 6 shows that the suboptimality of selected central nodes generally increases with the value of $K$, which is consistent with our theoretical expectation in Theorem 1. However, by comparing Fig. 6 with Fig. 4 , we notice that this suboptimality may also be diminished if the value of $K$ is appropriately selected to reflect the heterogeneity of network contact pattern. For example, Fig. $4 \mathrm{~b}$ shows that the metric values of five nodes are much higher than those of other nodes in the Infocom06 trace. Correspondingly, the suboptimality of distributed NCL selection can be reduced as shown in Fig. $6 \mathrm{~b}$ when the value of $K$ is changed from 3 to 5. Similar cases are also found in all the other traces.

\section{Caching Scheme}

In this section, we present our cooperative caching scheme. Our basic idea is to intentionally cache data at a set of NCLs, which can be promptly accessed by other nodes. Our scheme consists of the following three components:

1. When a data source generates data, it pushes data to central nodes of NCLs, which are prioritized to cache data. One copy of data is cached at each NCL. If the caching buffer of a central node is full, another node near the central node will cache the data. Such

3. We have $I_{j}=j$ for $\forall j$ if the optimal central nodes are selected. decisions are automatically made based on buffer conditions of nodes involved in the pushing process.

2. A requester multicasts a query to central nodes of NCLs to pull data, and a central node forwards the query to the caching nodes. Multiple data copies are returned to the requester, and we optimize the tradeoff between data accessibility and transmission overhead by controlling the number of returned data copies.

3. Utility-based cache replacement is conducted whenever two caching nodes contact and ensures that popular data are cached nearer to central nodes. We generally cache more copies of popular data to optimize the cumulative data access delay. We also probabilistically cache less popular data to ensure the overall data accessibility.

\subsection{Caching Location}

Whenever a node $S$ generates new data, $S$ pushes the data to NCLs by sending a data copy to each central node representing a NCL. We use the opportunistic path weight to the central node as relay selection metric for such data forwarding, and a relay forwards data to another node with a higher metric than itself. This "Compare-and-Forward" strategy has been widely used in the literature [10], [9] for efficient data forwarding. According to Definition 1 on opportunistic path, this strategy probabilistically ensures that each forwarding reduces the remaining delay for data to be delivered to the central node.

For newly generated data, the initial caching locations are automatically determined during the forwarding process based on node buffer conditions. The caching locations are then dynamically adjusted by cache replacement described in Section 5.4 according to query history. In general, data are forwarded to and cached at central nodes. This forwarding process only stops when the caching buffer of the next relay is full, ${ }^{4}$ and data are cached at the current relay in such cases. In other words, during the data forwarding process toward central nodes, relays carrying data are considered as temporal caching locations of the data.

Such determination of caching location is illustrated in Fig. 7, where the solid lines indicate opportunistic contacts used to forward data, and the dashed lines indicate data forwarding stopped by node buffer constraint. Central node $C_{1}$ is able to cache data, but data copies to $C_{2}$ and $C_{3}$ are

4. Since the data are newly generated and has not been requested yet, no cache replacement is necessary at the relay. 


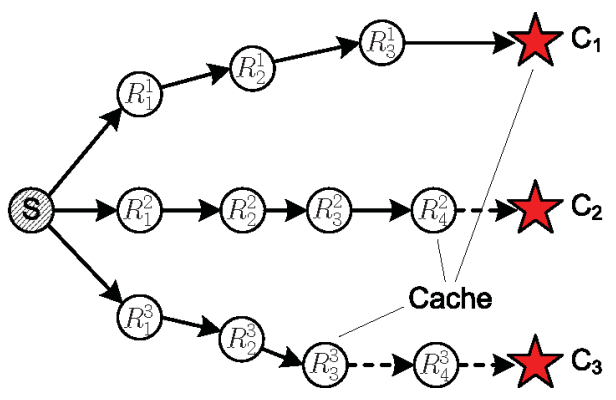

Fig. 7. Determining caching location at NCLs.

stopped and cached at relays $R_{4}^{2}$ and $R_{3}^{3}$, respectively, because neither $C_{2}$ nor $R_{4}^{3}$ has enough buffer to cache data. Note that the caching location at a NCL may not be the contacted neighbor of a central node, like the case of nodes $R_{3}^{3}$ in Fig. 7 .

From this strategy, it is easy to see that the set of caching nodes at each NCL forms a connected subgraph of the network contact graph at any time during data access. This property essentially facilitates the delivery of user queries to the caching nodes, which is described in Section 5.2.

\subsection{Queries}

We assume that any node may request data, and hence, data requesters are randomly distributed in the network. A requester multicasts a query with a finite time constraint to all the central nodes to pull data, and existing multicast schemes in DTNs [20] can be exploited for this purpose.

After having received the query, a central node immediately replies to the requester with data if it is cached locally. ${ }^{5}$ Otherwise, it broadcasts the query to the nodes nearby. This process is illustrated in Fig. 8. While the central node $C_{1}$ is able to return the cached data to $R$ immediately, the caching nodes $A$ and $B$ only reply to $R$ after they receive the query from central nodes $C_{2}$ and $C_{3}$, respectively. The query broadcast finishes when query expires. Each caching node at NCLs maintains up-to-date information about query history, which is used in Section 5.4 for cache replacement.

\subsection{Probabilistic Response}

As shown in Fig. 8, multiple data copies are replied to the requester from NCLs to ensure that the requester receives data before query expires. However, only the first data copy received by the requester is useful, and all the others are essentially useless and waste network resources. The major challenge for solving this problem arises from the intermittent network connectivity in DTNs. First, it is difficult for caching nodes to promptly communicate with each other, and hence, the optimal number of data copies returned to the requester cannot be determined in advance. Second, a relay carrying a data copy does not know the locations of other data copies being returned, and therefore cannot determine whether the requester has received data.

In this section, we propose a probabilistic scheme to address these challenges and optimize the tradeoff between data accessibility and transmission overhead. Our basic idea

5. Particularly, if a caching node is selected as the relay during multicasting of a query, it directly sends the cached data to the requester, without forwarding the query to the central node.

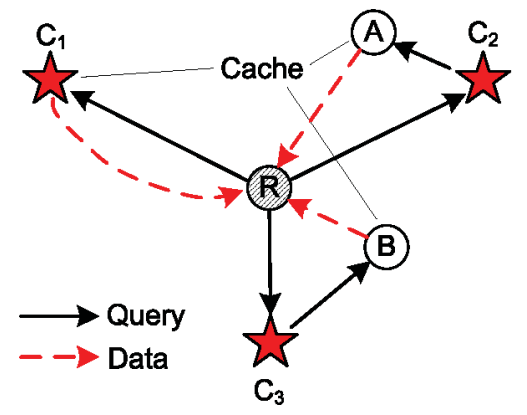

Fig. 8. Pulling data from the NCLs.

is that, having received the query, a caching node probabilistically decides whether to return the cached data to the requester. Different strategies are used for this decision, according to the availability of network contact information.

We assume that a query is generated with a time constraint $T_{q}$, and it takes $t_{0}<T_{q}$ for it to be forwarded from requester $R$ to caching node $C$. If $C$ knows the information about the shortest opportunistic paths to all the nodes in the network, $C$ can determine whether to reply data to $R$ with the probability $p_{C R}\left(T_{q}-t_{0}\right)$. According to (2), $p_{C R}\left(T_{q}-t_{0}\right)$ indicates the probability that data can be transmitted from $C$ to $R$ within the remaining time $T_{q}-t_{0}$ for responding to the query.

Otherwise, $C$ only maintains information about shortest opportunistic paths to central nodes, and it is difficult for $C$ to estimate the data transmission delay to $R$. Instead, the probability for deciding data response is calculated only based on the remaining time $T_{q}-t_{0}$. This probability should be proportional to $T_{q}-t_{0}$, and we calculate this probability as a Sigmoid function $p_{R}(t)$, where $p_{R}\left(T_{q}\right)=$ $p_{\max } \in(0,1]$ and $p_{R}(0)=p_{\min } \in\left(p_{\max } / 2, p_{\max }\right)$. This function is written as

$$
p_{R}(t)=\frac{k_{1}}{1+e^{-k_{2} \cdot t}},
$$

where $k_{1}=2 p_{\min }, k_{2}=\frac{1}{T_{q}} \cdot \ln \left(\frac{p_{\max }}{2 p_{\min }-p_{\max }}\right)$. The quantities $p_{\max }$ and $p_{\min }$ in (10) are user-specified parameters of the maximum and minimum response probabilities. As an example, the sigmoid function with $p_{\min }=0.45, p_{\max }=0.8$, and $T_{q}=10$ hours is shown in Fig. 9 .

\subsection{Cache Replacement}

For each data item in the network, the locations where it is cached are dynamically adjusted via cache replacement. This replacement is based on data popularity, and generally

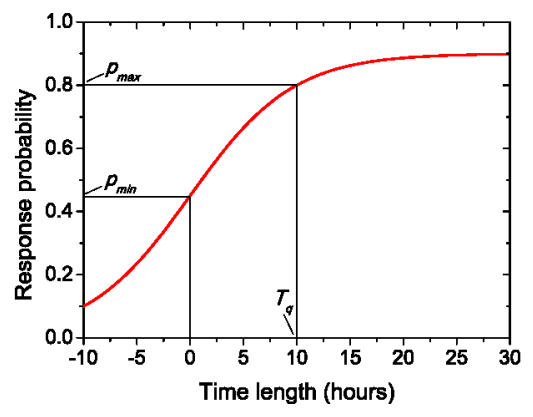

Fig. 9. Probability for deciding data response. 
places popular data nearer to the central nodes of NCLs. Traditional cache replacement strategies such as LRU, which removes the least-recently-used data from cache when new data are available, are ineffective due to its oversimplistic consideration of data popularity. Greedy-Dual-Size [6] calculates data utility by considering data popularity and size simultaneously, but cannot ensure optimal selection of cached data. We improve previous work by proposing a probabilistic cache replacement strategy, which appropriately selects the data to be cached and heuristically balances between the cumulative data accessibility and access delay.

\subsubsection{Data Popularity}

The popularity of a data item is probabilistically estimated based on the past $k$ requests to this data during time period $\left[t_{1}, t_{k}\right]$. We assume that such occurrences of data requests follow a Poisson distribution with the parameter $\lambda_{d}=k /\left(t_{k}-t_{1}\right)$, and data popularity is defined as the probability that this data will be requested again in the future before data expires. If data $d_{i}$ expire at time $t_{e}$, its popularity is $w_{i}=1-e^{-\lambda_{d} \cdot\left(t_{e}-t_{k}\right)}$. To calculate $w_{i}$, a node only needs to recursively maintain two time values about the past occurrences of data requests, and therefore will only incur negligible space overhead.

\subsubsection{Basic Strategy}

Cache replacement opportunistically occurs whenever two caching nodes $A$ and $B$ contact. The two nodes exchange their cached data to optimize the cumulative data access delay. ${ }^{6}$ We collect the cached data at both nodes into a selection pool $\mathbb{S}=\left\{d_{1}, \ldots, d_{n}\right\}$, and formulate cache replacement as follows:

$$
\begin{aligned}
\max & \sum_{i=1}^{n} x_{i} u_{i}+\sum_{j=1}^{n} y_{j} v_{j} \\
\text { s.t. } & \sum_{i=1}^{n} x_{i} s_{i} \leq S_{A}, \sum_{j=1}^{n} y_{j} s_{j} \leq S_{B} \\
& x_{i}+y_{i} \leq 1, \text { for } \forall i \in[1, n],
\end{aligned}
$$

where $x_{i}, y_{i} \in[0,1]$ indicate whether data $d_{i}$ are cached at node $A$ and $B$ after replacement, respectively. $s_{i}$ indicates size of data $d_{i}$, and $S_{A}$ and $S_{B}$ are the buffer sizes of $A$ and $B$. $u_{i}=w_{i} \cdot p_{A}$ and $v_{i}=w_{i} \cdot p_{B}$ indicate the utility of data $d_{i}$ at $A$ and $B$ to cumulative caching performance, where $w_{i}$ is popularity of data $d_{i} ; p_{A}$ and $p_{B}$ are the weights of the shortest opportunistic path to the corresponding central node.

This formulation places popular data to caching nodes near the central nodes. It is NP-hard because the standard 01 knapsack problem can reduce to this problem. We propose a heuristic to approximate the solution of this problem.

Without loss of generality, we assume that $p_{A}>p_{B}$, and node $A$ is prioritized to select its data to cache from $\mathbf{S}$ by solving the following problem extracted from (11):

$$
\begin{aligned}
& \max \sum_{i=1}^{n} x_{i} u_{i} \\
& \text { s.t. } \sum_{i=1}^{n} x_{i} s_{i} \leq S_{A} .
\end{aligned}
$$

6. Since nodes only exchange data when they contact, it is unnecessary for a caching node to actively remove obsolete data from its local cache.

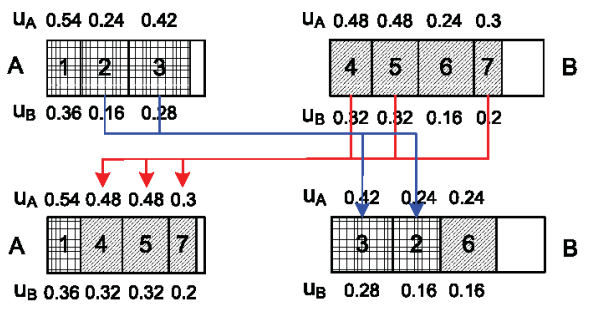

(a) Normal cache replacement

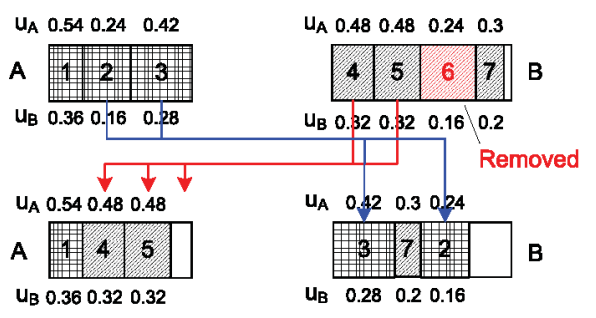

(b) Possible data removal

Fig. 10. Cache replacement.

Afterwards, node $B$ selects data to cache from the remaining part of $\mathbb{S}$ by solving a similar problem to (12). Since $S_{A}$ and $s_{i}$ in (12) are usually integers in numbers of bytes, this problem can be solved in pseudopolynomial time $O\left(n \cdot S_{A}\right)$ using a dynamic programming approach [26].

This replacement process is illustrated in Fig. 10, where initially node $A$ caches data $d_{1}, d_{2}$, and $d_{3}$, and node $B$ caches data $d_{4}, d_{5}, d_{6}$, and $d_{7}$. The two nodes exchange and replace their cached data upon contact, based on the data utility values listed as $u_{A}$ and $u_{B}$. As shown in Fig. 10a, since $p_{A}>p_{B}$, node $A$ generally caches the popular data $d_{4}, d_{5}$, and $d_{7}$, and leaves data $d_{2}$ and $d_{3}$ with lower popularity to node $B$.

In cases of limited cache space, some cached data with lower popularity may be removed from caching buffer. In Fig. 10b, when the sizes of caching buffer of nodes $A$ and $B$ decrease, $A$ does not have enough buffer to cache data $d_{7}$, which is instead cached at node $B$. Data $d_{6}$ with the lowest popularity will then be removed from cache because neither node $A$ nor $B$ has enough space to cache it.

\subsubsection{Probabilistic Data Selection}

The aforementioned removal of cached data essentially prioritizes popular data during cache replacement, but may impair the cumulative data accessibility. The major reason is that according to our network modeling in Section 3.2, the data accessibility does not increase linearly with the number of cached data copies in the network. More specifically, the data accessibility will increase considerably if the number of cached data copies increases from 1 to 2, but the benefit will be much smaller if the number increases from 10 to 11 . In such cases, for the example shown in Fig. 10b, caching $d_{1}$ at node $A$ may be ineffective because the popular $d_{1}$ may already be cached at many other places in the network. In contrast, removing $d_{6}$ out from the cache of node $B$ may greatly impair the accessibility of $d_{6}$ because there may be only few cached copies of $d_{6}$ due to its lower popularity.

In other words, the basic strategy of cache replacement only optimizes the cumulative data access delay within the 
local scope of the two caching nodes in contact. Such optimization at the global scope is challenging in DTNs due to the difficulty of maintaining knowledge about the current number of cached data copies in the network, and we instead propose a probabilistic strategy to heuristically control the number of cached data copies at the global scope.

The basic idea is to probabilistically select data to cache when the problem in (12) is solved by a dynamic programming approach. More specifically, if data $d_{i}$ are selected by the dynamic programming algorithm, it has probability $u_{i}$ to be cached at node $A$. This algorithm is described in detail in Algorithm 1, where $\operatorname{GetMax}\left(\mathbb{S}, S_{A}\right)$ calculates the maximal possible value of the items in the knapsack via dynamic programming, and $\operatorname{SelectData}\left(d_{i_{\max }}\right)$ determines whether to select data $d_{i_{\max }}$ to cache at node $A$ by conducting a Bernoulli experiment with probability $u_{i_{\max }}$. Such probabilistic selection may be iteratively conducted multiple times to ensure that the caching buffer is fully utilized. By proposing this probabilistic strategy, we still prioritize the popular data with higher utility during the caching decision, but also enable the data with less popularity to have nonnegligible chance to be cached.

Algorithm 1. Probabilistic Data Selection at node $A$ among the data set $\mathbb{S}$.

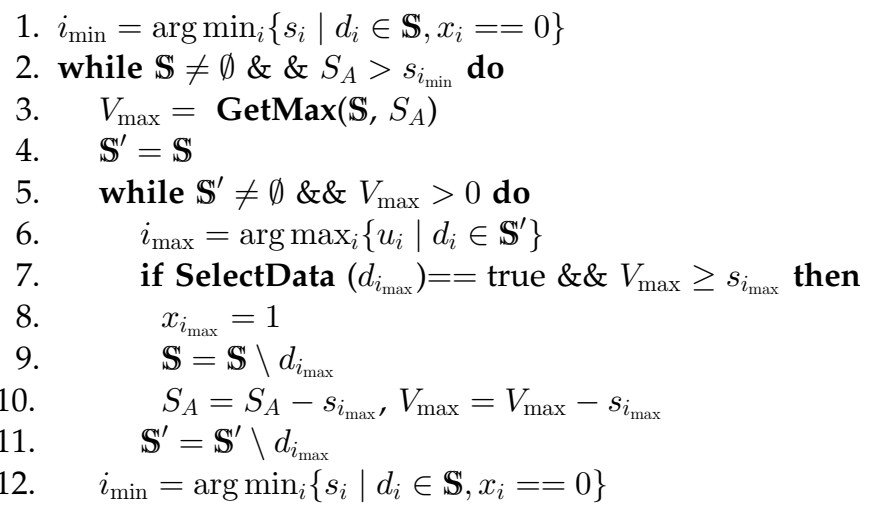

\subsection{Discussions}

In summary, data access delay of our scheme consists of three parts: 1) the time for query to be transmitted from requester to central nodes; 2 ) the time for central nodes to broadcast query to caching nodes; 3 ) the time for the cached data to be returned to requester.

Data access delay is closely related to the number $(K)$ of NCLs. When $K$ is small, the average distance from a node to the NCLs is longer, which makes the first and third parts of the delay bigger. Meanwhile, since the total amount of data being cached in the network is small, data are more likely to be cached near to the central nodes, and the second part of the delay can be short. In contrast, if $K$ is large, the metric values of some central nodes may not be high, and hence caching at the corresponding NCLs may be less efficient. Moreover, when the node buffer constraint is tight, a caching node may be shared by multiple NCLs. The NCLs with lower caching effectiveness may disturb the caching decision of other NCLs and furthermore impair the caching performance.

It is clear that the number $(K)$ of NCLs is vital to the performance of our caching scheme. In Section 7.4, we will

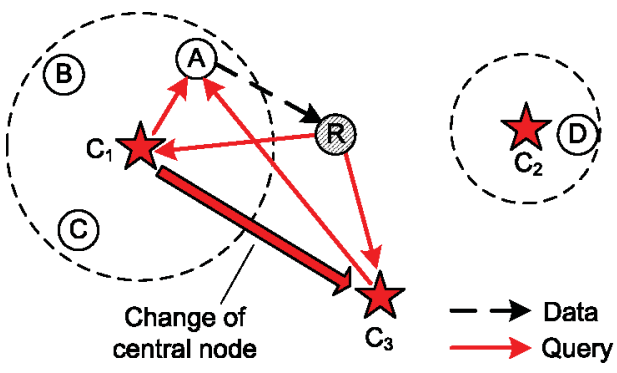

Fig. 11. NCL load balancing.

experimentally investigate the impact of different values of $K$ on the caching performance in more details.

\section{NCL LOAD BALANCING}

From the caching scheme proposed in Section 5, we can see that the central nodes play vital roles in cooperative caching in DTNs. First, the central nodes cache the most popular data in the network and respond to the frequent queries for these data. Second, the central nodes are also responsible for broadcasting all the queries they receive to other caching nodes nearby. However, such functionality may quickly consume the local resources of central nodes that include their battery life and local memory. In addition, we would like our caching schemes to be resilient to failures of central nodes. In this section, we focus on addressing this challenge, and propose methods that efficiently migrate the functionality of central nodes to other nodes in cases of failures or resource depletion. In general, the methods we present in this section can be used to adjust the deployment of central nodes at runtime, such as adding or removing central nodes according to up-to-date requirements on caching performance.

\subsection{Selecting the New Central Node}

When a central node fails or its local resources are depleted, another node is selected as a new central node. Intuitively, the new central node should be the one with the highest NCL selection metric value among the current noncentral nodes in the network. However, such selection may degrade the caching performance as illustrated in Fig. 11. When the local resources of central node $C_{1}$ are depleted, its functionality is taken over by $C_{3}$. Since $C_{3}$ may be far away from $C_{1}$, the queries broadcasted from $C_{3}$ may take a long time to reach the caching nodes $A$, and hence reduce the probability that the requester $R$ receives data from $A$ on time. From Fig. 11, it is easy to see that such performance degradation is caused by the existing data being cached at nodes near $C_{1}$.

In this case, the distance between the new central node and $C_{1}$ should also be taken into account. More specifically, with respect to the original central node $j$, we define the metric $C_{i}^{j}$ for a node $i$ to be selected as the new central node as

$$
C_{i}^{j}=C^{i} \cdot p_{i j}(T),
$$

where $C_{i}$ is the original NCL selection metric defined in Section 4 and $p_{i j}(T)$ is the weight of the shortest opportunistic path between node $i$ and $j$ defined in (2).

In practice, an existing central node $j$ is responsible for selecting the new central node $i$ when its local resources are 
depleted according to the metric defined in (13); node $j$ also broadcasts a notification to the entire network indicating the new central node. If node $j$ is unable to do so due to sudden failure, another node in contact with $j$ will be responsible for such selection and notification. To realize this, node $j$ designates one of its contacted neighbors with the maximum battery life as its "backup," and synchronizes this backup node with all the information that $j$ has regarding the contact capabilities of other nodes. As a result, when node $j$ suddenly fails due to resource depletion, this backup node will be responsible for selecting the new central node.

\subsection{Adjustment of Caching Locations}

After a new central node is selected, the data cached at the NCL represented by the original central node needs to be adjusted correspondingly, so as to optimize the caching performance. For example in Fig. 11, after the functionality of central node $C_{1}$ has been migrated to $C_{3}$, the nodes $A, B$, and $C$ near $C_{1}$ are not considered as good locations for caching data anymore. Instead, the data cached at these nodes needs to be moved to other nodes near $C_{3}$.

This movement is achieved via cache replacement when caching nodes opportunistically contact each other. Each caching node at the original NCL recalculates the utilities of its cached data items with respect to the newly selected central node. In general, these data utilities will be reduced due to the changes of central nodes, and this reduction moves the cached data to the appropriate caching locations that are nearer to the newly selected central node.

Changes in central nodes and subsequent adjustment of caching locations inevitably affect caching performance as shown in Fig. 11. However, this performance degradation will be gradually eliminated over time by the opportunistic cache replacement. In Section 7, we will furthermore evaluate such impact, in practice, on realistic DTN traces.

\section{Performance Evaluation}

We evaluate the performance of our proposed caching scheme by comparing it with the following schemes:

- No Cache, where caching is not used for data access and each query is only responded by data source.

- Random Cache, in which every requester caches the received data to facilitate data access in the future.

- CacheData [35], which is proposed for cooperative caching in wireless ad hoc networks, and lets each relay in DTNs cache the pass-by data based on their popularity.

- Bundle Cache [27], which packs network data as bundles and makes caching decision on pass-by data by considering the node contact pattern in DTNs, so as to minimize the average data access delay.

Cache replacement algorithms are proposed in CacheData and Bundle Cache, and will also be used in our evaluations. For Random Cache, LRU is used for cache replacement. The following metrics are used for evaluations. Each simulation is repeated multiple times with randomly generated data and queries for statistical convergence:

- Successful ratio, the ratio of queries being satisfied with the requested data. This ratio evaluates the
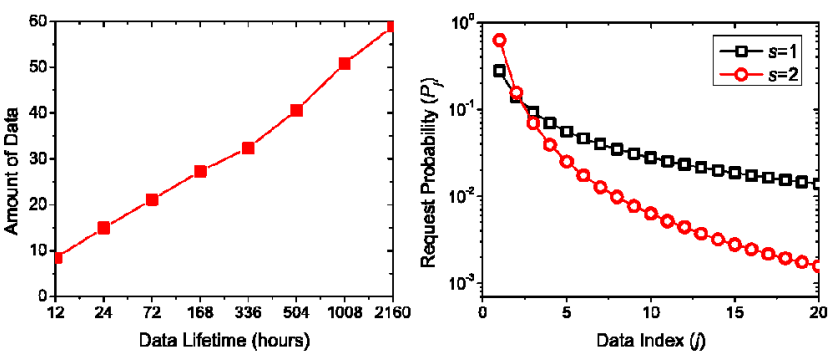

(a) Amount of network data (b) Data request probabilities

Fig. 12. Experiment setup.

coverage of data access provided by our proposed caching schemes.

- Data access delay, the average delay for getting responses to queries.

- Caching overhead, the average number of data copies being cached in the network. ${ }^{7}$

\subsection{Experiment Setup}

Our performance evaluations are performed on the Infocom06 and MIT Reality traces. In all the experiments, central nodes representing NCLs are globally selected before data and queries are generated. The first half of the trace is used as warm-up period for the accumulation of network information and subsequent NCL selection, and all the data and queries are generated during the second half of trace.

\subsubsection{Data Generation}

Each node periodically checks whether it has generated data which has not expired yet. If not, the node determines whether to generate new data with probability $p_{G}$. Each generated data have finite lifetime uniformly distributed in range $[0.5 T, 1.5 T]$, and the period for data generation decision is also set as $T$. In our evaluations, we fix $p_{G}=0.2$, and the amount of data in the network is, hence, controlled by $T$, as illustrated in Fig. 12a for the MIT Reality trace. Similarly, data size is uniformly distributed in range $\left[0.5 s_{\text {avg }}, 1.5 s_{\text {avg }}\right]$, and caching buffers of nodes are uniformly distributed in range $[200,600 \mathrm{Mb}] . s_{\text {avg }}$ is adjusted to simulate different node buffer conditions.

Note that in this section, we compare the performance of our proposed schemes with the existing work. When $T$ is large, indicating long intercontact time among mobile nodes in the network, our experimental setup increases the data lifetime accordingly. In this way, we ensure nonnegligible caching performance in the network and furthermore comprehensive performance comparisons. We could reasonably infer that the comparison results we have in this section will still hold, when the average intercontact time in the network is reduced and enables efficient access on data with shorter lifetime.

\subsubsection{Query Pattern}

Queries are randomly generated at all nodes, and each query has a finite time constraint $T / 2$. In particular, every time $T / 2$,

7. We consider the overhead of maintaining node contact information as negligible because only the pairwise contact rates are maintained for calculating NCL selection metric, as described in Section 4.1. 


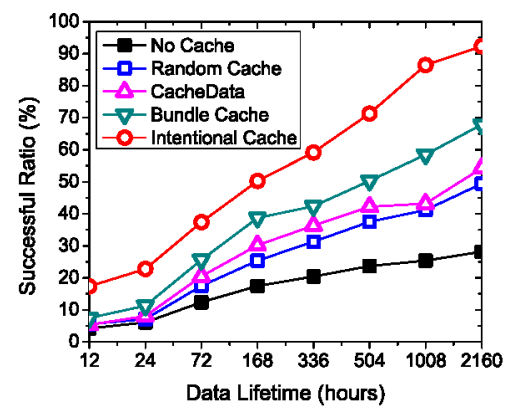

(a) Successful ratio

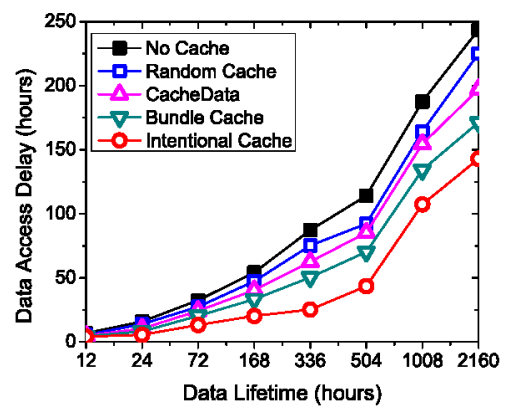

(b) Data access delay

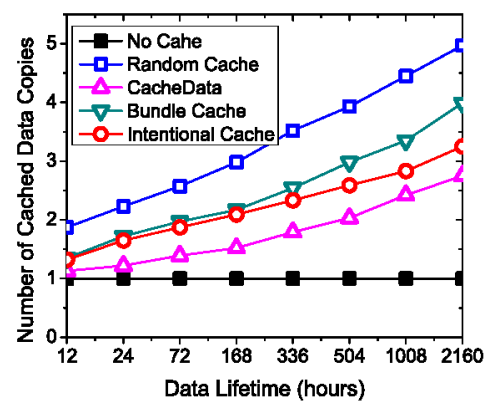

(c) Caching overhead

Fig. 13. Performance of data access with different data lifetime.

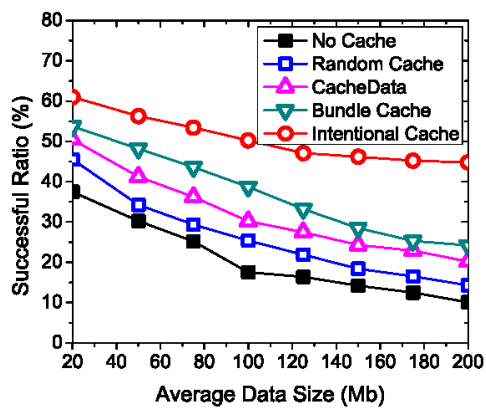

(a) Successful ratio

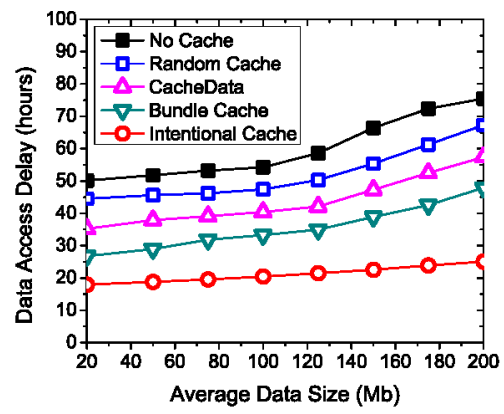

(b) Data access delay

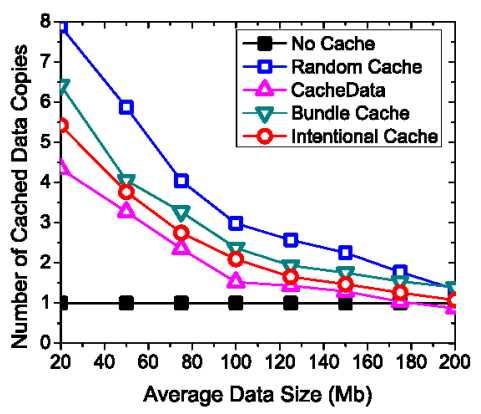

(c) Caching overhead

Fig. 14. Performance of data access with different node buffer conditions.

each node independently determines whether to generate a query for data $j$ with probability $P_{j}$. We assume that query pattern follows a Zipf distribution that has been proved to describe the query pattern of web data access [3]. As a result, letting $M$ be the number of data items in the network, we have $P_{j}=\frac{1}{j^{s}} /\left(\sum_{i=1}^{M} \frac{1}{i^{s}}\right)$, where $s$ is an exponent parameter. Values of $P_{j}$ with different $s$ are shown in Fig. 12b.

\subsection{Caching Performance}

Caching performance of our scheme is evaluated using MIT Reality trace. The number $(K)$ of NCLs is set to 8 and query pattern follows a Zipf distribution with $s=1$. By default, $T=1$ week and $s_{\text {avg }}=100 \mathrm{Mb}$. These two parameters are then adjusted for different performance evaluation purposes.

The simulation results with different values of $T$ are shown in Fig. 13. The successful ratio of data access is mainly restrained by $T$ itself. When $T$ increases from 12 hours to three months, the successful ratio of all schemes is significantly improved because data have more time to be delivered to requesters before expiration. Since the selected NCLs are efficient in communicating with other nodes, our proposed intentional caching scheme achieves much better successful ratio and delay of data access. As shown in Figs. 13a and 13b, the performance of our scheme is 200 percent better than that of NoCache, and also exhibits 50 percent improvement over BundleCache, where nodes also incidentally cache pass-by data. Comparatively, RandomCache is ineffective due to the random distribution of requesters in the network, and CacheData is also inappropriate for DTNs due to the difficulty of maintaining query history.
Meanwhile, Fig. 13c shows that our scheme only requires moderate cache size, which is much lower than that required by RandomCache and BundleCache, especially when $T$ is large. RandomCache consumes the largest caching buffer, such that each data have five cached copies when $T$ increases to three months. The major reason is that each requester blindly caches any received data until its buffer is filled up. CacheData consumes 30 percent less buffer than our scheme, but also leaves a lot of data uncached that impairs data access performance. We notice that caching overhead in our scheme also includes the transmission and storage cost when queries and data are transmitted between requesters and caching nodes, and realize that such cost is proportional to data access delay during which data are carried by relays. Hence, the cost-effectiveness of our scheme is also supported by Fig. 13b.

We also evaluated data access performance with different node buffer conditions by adjusting $s_{\text {avg }}$ and the results are shown in Fig. 14. When data size becomes larger, less data can be cached as shown in Fig. 14c, and data access performance is hence reduced. In Figs. 14a and $14 \mathrm{~b}$, when $s_{\text {avg }}$ increases from 20 to $200 \mathrm{Mb}$, the successful ratio of our scheme decreases from 60 to 45 percent, and data access delay increases from 18 to 25 hours. However, the performances of other schemes even decrease much faster, and the advantage of our scheme becomes even larger when node buffer constraint is tight. This is mainly due to the intelligent cache replacement strategy used in our scheme, which ensures that the most appropriate data are cached. 


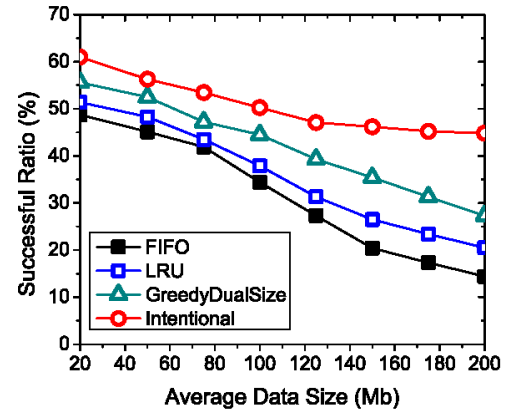

(a) Successful ratio

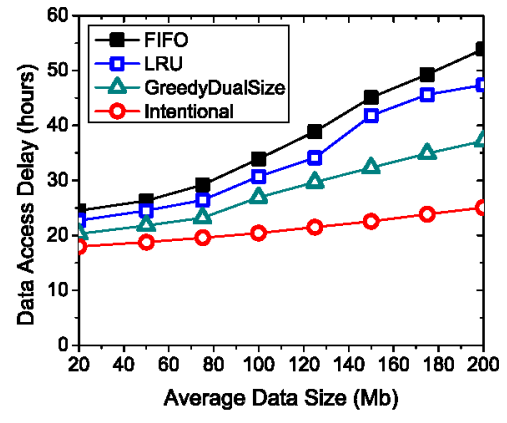

(b) Data access delay

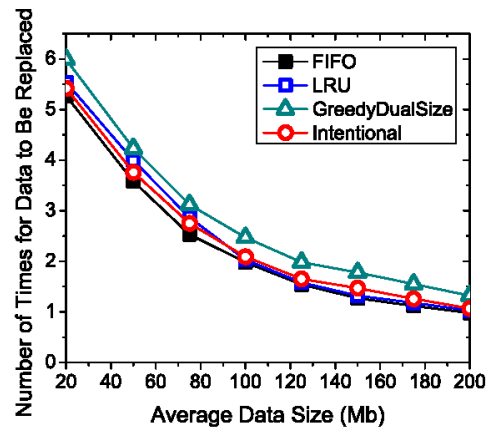

(c) Cache Replacement overhead

Fig. 15. Performance of data access with different cache replacement strategies.

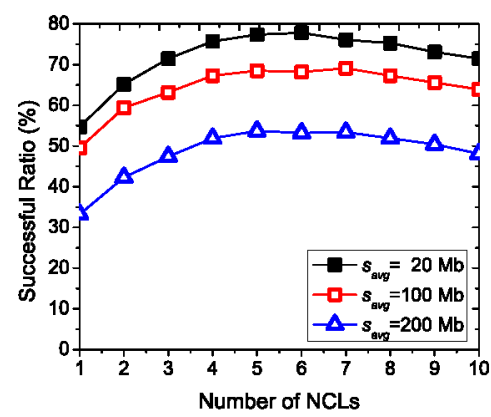

(a) Successful ratio

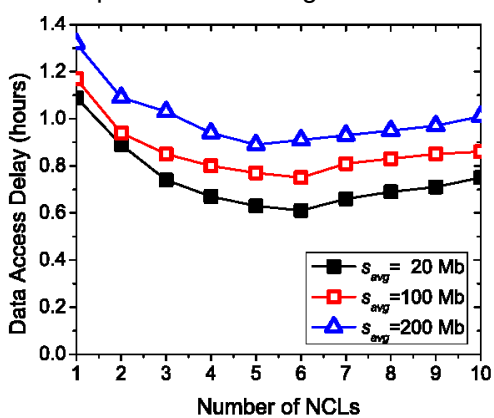

(b) Data access delay

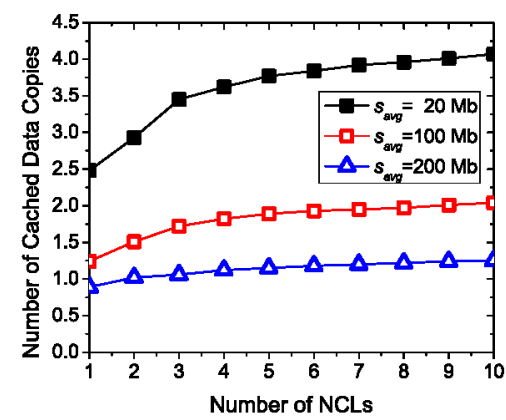

(c) Caching overhead

Fig. 16. Performance of data access with different number of NCLs.

\subsection{Effectiveness of Cache Replacement}

Our proposed cache replacement strategy in Section 5.4 is compared with the traditional replacement strategies including FIFO and LRU. It is also compared with Greedy-Dual-Size, which is widely used in web caching.

We use MIT Reality trace for such evaluation, and set $T$ as one week. The results are shown in Fig. 15. FIFO and LRU leads to poor data access performance due to improper consideration of data popularity. In Fig. 15a, when data size is small and node buffer constraint is not tight, cache replacement will not be frequently conducted. Hence, the successful ratio of traditional strategies is only 10-20 percent lower than that of our scheme. However, when data size becomes larger, these strategies do not always select the most appropriate data to cache, and the advantage of our scheme rises to over 100 percent when $s_{\text {avg }}=200 \mathrm{Mb}$. Data access delay of FIFO and LRU also becomes much longer when $s_{\text {avg }}$ increases as shown in Fig. 15b. Greedy-Dual-Size performs better than FIFO and LRU due to consideration of data popularity and size, but it is unable to ensure optimal cache replacement decision.

In Fig. 15c, we also compared the overhead of those strategies, which is the amount of data exchanged for cache replacement. Since cache replacement is only conducted locally between mobile nodes in contact, there are only slight differences of this overhead among different strategies. Greedy-Dual-Size makes the caching nodes exchange a bit more data, but this difference is generally negligible.

\subsection{Number of NCLs}

In this section, we investigate the impact of different numbers $(K)$ of NCLs on data access performance using
Infocom06 trace. We set $T=3$ hours and all the other parameters remain the same as in Section 7.2.

The simulation results are shown in Fig. 16. When $K$ is small, it takes longer to forward queries and data between requesters and caching nodes, and hence data access performance is reduced. This reduction is particularly significant when $K<3$. As shown in Figs. 16a and 16b, when $K$ is reduced from 2 to 1 , the delivery ratio decreases by 25 percent, and the data access delay increases by 30 percent. In contrast, when $K$ is large, further increase of $K$ will not improve data access performance because the newly selected central nodes are essentially not good at communicating with other nodes in the network. Meanwhile, as shown in Fig. 16c, when $K$ is small, increasing $K$ will consume considerably more buffer space for caching. However, this increase is negligible when $K$ is large or node buffer constraint is tight.

In summary, when node buffer constraint is tight, smaller $K$ is helpful to provide acceptable caching performance with lower overhead. However, too large $K$ will not provide any extra benefit, and may even impair the performance. From Fig. 16, we conclude that $K=5$ is the best choice for Infocom06 trace, which is consistent with the result of trace-based validation shown in Fig. $4 \mathrm{~b}$.

\subsection{Impact of NCL Load Balancing}

In this section, we evaluate the impact of NCL load balancing scheme proposed in Section 6 on caching performance. According to the evaluation results in Section 7.4, we set $K=8$ for the MIT Reality trace and $s_{\text {avg }}=100 \mathrm{Mb}$. 


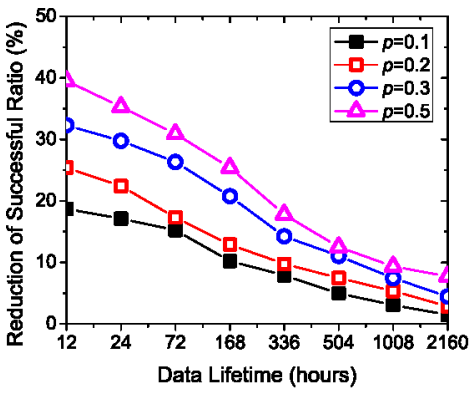

(a) Reduction of successful ratio

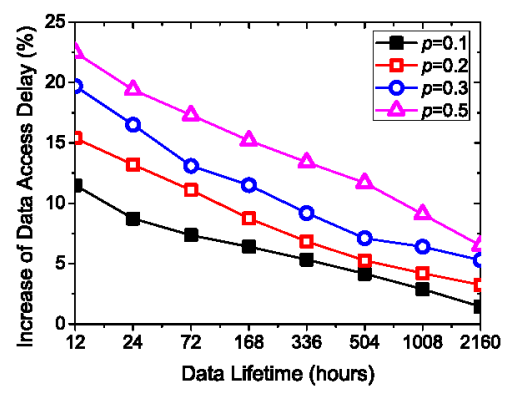

(b) Increase of data access delay

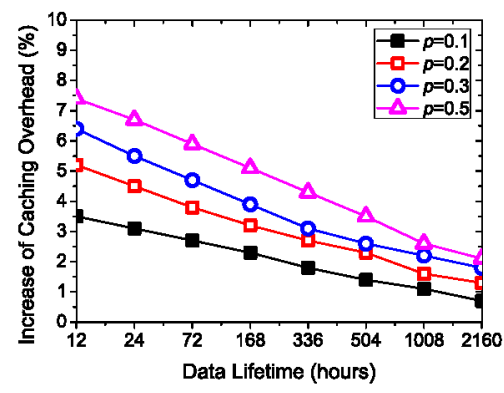

(c) Increase of caching overhead

Fig. 17. Impact of NCL load balancing on the caching performance.

Each central node periodically determines whether to migrate its functionality to another node with a fixed probability $p$. We set the period of making such decision to be 10 percent of the trace length, and the evaluation results with different values of $p$ on the MIT Reality trace are shown in Fig. 17. In general, when the central nodes change, the existing caching locations become inappropriate, and hence, the successful ratio of data access is reduced. As shown in Fig. 17a, such reduction can be up to 40 percent when the data lifetime is short, but will diminish significantly to 10 percent when there is longer time for the queries to be forwarded to the caching nodes. Fig. $17 \mathrm{~b}$ also shows that the data access delay increases accordingly. Moreover, since the cached data copies are opportunistically moved to more appropriate network locations after the change of central nodes, the caching overhead only slightly increases by less than 10 percent, as shown in Fig. 17c.

The impact of NCL load balancing is also determined by the frequency of the changes of central nodes. As shown in Fig. 17, the reduction of successful ratio of data access is sensitive to the value of $p$. Especially, when the data lifetime is short, larger value of $p$ significantly magnify the impact on the caching performance. In general, the impact of NCL load balancing on the caching performance is largely determined by the specific network condition and data access pattern.

\section{Conclusions}

In this paper, we propose a novel scheme to support cooperative caching in DTNs. Our basic idea is to intentionally cache data at a set of NCLs, which can be easily accessed by other nodes. We ensure appropriate NCL selection based on a probabilistic metric; our approach coordinates caching nodes to optimize the tradeoff between data accessibility and caching overhead. Extensive simulations show that our scheme greatly improves the ratio of queries satisfied and reduces data access delay, when being compared with existing schemes.

\section{ACKNOWLEDGMENTS}

This work was supported in part by Network Science CTA under grant W911NF-09-2-0053.

\section{REFERENCES}

[1] A. Balasubramanian, B. Levine, and A. Venkataramani, "DTN Routing as a Resource Allocation Problem," Proc. ACM SIGCOMM Conf. Applications, Technologies, Architectures, and Protocols for Computer Comm., pp. 373-384, 2007.

[2] C. Boldrini, M. Conti, and A. Passarella, "ContentPlace: SocialAware Data Dissemination in Opportunistic Networks," Proc. 11th Int'l Symp. Modeling, Analysis and Simulation of Wireless and Mobile Systems (MSWiM), pp. 203-210, 2008.

[3] L. Breslau, P. Cao, L. Fan, G. Phillips, and S. Shenker, "Web Caching and Zipf-Like Distributions: Evidence and Implications." Proc. IEEE INFOCOM, vol. 1, 1999.

[4] J. Burgess, B. Gallagher, D. Jensen, and B. Levine, "MaxProp Routing for Vehicle-Based Disruption-Tolerant Networks," Proc. IEEE INFOCOM, 2006

[5] H. Cai and D.Y. Eun, "Crossing over the Bounded Domain: From Exponential to Power-Law Inter-Meeting Time in MANET," Proc. ACM MobiCom, pp. 159-170, 2007.

[6] P. Cao and S. Irani, "Cost-Aware WWW Proxy Caching Algorithms," Proc. USENIX Symp. Internet Technologies and Systems, 1997.

[7] A. Chaintreau, P. Hui, J. Crowcroft, C. Diot, R. Gass, and J. Scott, "Impact of Human Mobility on Opportunistic Forwarding Algorithms," IEEE Trans. Mobile Computing, vol. 6, no. 6, pp. 606-620, June 2007

[8] P. Costa, C. Mascolo, M. Musolesi, and G. Picco, "Socially Aware Routing for Publish-Subscribe in Delay-Tolerant Mobile Ad Hoc Networks," IEEE J. Selected Areas in Comm., vol. 26, no. 5, pp. 748760, June 2008.

[9] E. Daly and M. Haahr, "Social Network Analysis for Routing in Disconnected Delay-Tolerant MANETs," Proc. ACM MobiHoc, 2007.

[10] H. Dubois-Ferriere, M. Grossglauser, and M. Vetterli, "Age Matters: Efficient Route Discovery in Mobile Ad Hoc Networks Using Encounter Ages," Proc. ACM MobiHoc, pp. 257-266, 2003.

[11] J. Eriksson, L. Girod, B. Hull, R. Newton, S. Madden, and H. Balakrishnan, "The Pothole Patrol: Using a Mobile Sensor Network for Road Surface Monitoring," Proc. ACM Sixth Ann. Int'l Conf. Mobile Systems, Applications and Services (MobiSys), 2008.

[12] V. Erramilli, A. Chaintreau, M. Crovella, and C. Diot, "Diversity of Forwarding Paths in Pocket Switched Networks," Proc. Seventh ACM SIGCOMM Conf. Internet Measurement (IMC), pp. 161-174, 2007.

[13] V. Erramilli, A. Chaintreau, M. Crovella, and C. Diot, "Delegation Forwarding," Proc. ACM MobiHoc, 2008.

[14] K. Fall, "A Delay-Tolerant Network Architecture for Challenged Internets," Proc. ACM SIGCOMM Conf. Applications, Technologies, Architectures, and Protocols for Computer Comm., pp. 27-34, 2003.

[15] L. Fan, P. Cao, J. Almeida, and A. Broder, "Summary Cache: A Scalable Wide-Area Web Cache Sharing Protocol," IEEE/ACM Trans. Networking, vol. 8, no. 3, pp. 281-293, June 2000.

[16] M. Fiore, F. Mininni, C. Casetti, and C.F. Chiasserini, "To Cache or Not to Cache?" Proc. IEEE INFOCOM, pp. 235-243, 2009.

[17] W. Gao and G. Cao, "On Exploiting Transient Contact Patterns for Data Forwarding in Delay Tolerant Networks," Proc. IEEE Int'l Conf. Network Protocols (ICNP), pp. 193-202, 2010. 
[18] W. Gao and G. Cao, "User-Centric Data Dissemination in Disruption Tolerant Networks," Proc. IEEE INFOCOM, 2011.

[19] W. Gao, G. Cao, A. Iyengar, and M. Srivatsa, "Supporting Cooperative Caching in Disruption Tolerant Networks," Proc. Int'l Conf. Distributed Computing Systems (ICDCS), 2011.

[20] W. Gao, Q. Li, B. Zhao, and G. Cao, "Multicasting in Delay Tolerant Networks: A Social Network Perspective," Proc. ACM MobiHoc, pp. 299-308, 2009.

[21] Y. Huang, Y. Gao, K. Nahrstedt, and W. He, "Optimizing File Retrieval in Delay-Tolerant Content Distribution Community," Proc. IEEE Int'l Conf. Distributed Computing Systems (ICDCS), pp. 308-316, 2009.

[22] P. Hui, J. Crowcroft, and E. Yoneki, "Bubble Rap: Social-Based Forwarding in Delay Tolerant Networks," Proc. ACM MobiHoc, 2008.

[23] S. Ioannidis, L. Massoulie, and A. Chaintreau, "Distributed Caching over Heterogeneous Mobile Networks," Proc. ACM SIGMETRICS Int'l Conf. Measurement and Modeling of Computer Systems, pp. 311-322, 2010.

[24] V. Lenders, G. Karlsson, and M. May, "Wireless Ad Hoc Podcasting," Proc. IEEE Fourth Ann. Comm. Soc. Conf. Sensor, Mesh and Ad Hoc Comm. and Networks (SECON), pp. 273-283, 2007.

[25] F. Li and J. Wu, "MOPS: Providing Content-Based Service in Disruption-Tolerant Networks," Proc. Int'l Conf. Distributed Computing Systems (ICDCS), pp. 526-533, 2009.

[26] S. Martello and P. Toth, Knapsack Problems: Algorithms and Computer Implementations. John Wiley \& Sons, 1990.

[27] M.J. Pitkanen and J. Ott, "Redundancy and Distributed Caching in Mobile DTNs," Proc. ACM/IEEE Second Workshop Mobility in the Evolving Internet Architecture (MobiArch), 2007.

[28] I. Psaras, L. Wood, and R. Tafazolli, "Delay-/Disruption-Tolerant Networking: State of the Art and Future Challenges," technical report, Univ. of Surrey, 2010.

[29] J. Reich and A. Chaintreau, "The Age of Impatience: Optimal Replication Schemes for Opportunistic Networks," Proc. ACM Fifth Int'l Conf. Emerging Networking Experiments and Technologies (CoNEXT), pp. 85-96, 2009.

[30] S.M. Ross, Introduction to Probability Models. Academic, 2006

[31] T. Spyropoulos, K. Psounis, and C. Raghavendra, "Spray and Wait: An Efficient Routing Scheme for Intermittently Connected Mobile Networks," Proc. ACM SIGCOMM Workshop Delay-Tolerant Networking, pp. 252-259, 2005.

[32] T. Spyropoulos, K. Psounis, and C. Raghavendra, "Efficient Routing in Intermittently Connected Mobile Networks: The Single-Copy Case," IEEE/ACM Trans. Networking, vol. 16, no. 1, pp. 63-76, Feb. 2008

[33] B. Tang, H. Gupta, and S.R. Das, "Benefit-Based Data Caching in Ad Hoc Networks," IEEE Trans. Mobile Computing, vol. 7, no. 3, pp. 289-304, Mar. 2008.

[34] A. Vahdat and D. Becker, "Epidemic Routing for Partially Connected Ad Hoc Networks," Technical Report CS-200006, Duke Univ., 2000.

[35] L. Yin and G. Cao, "Supporting Cooperative Caching in Ad Hoc Networks," IEEE Trans. Mobile Computing, vol. 5, no. 1, pp. 77-89, Jan. 2006.

[36] E. Yoneki, P. Hui, S. Chan, and J. Crowcroft, "A Socio-Aware Overlay for Publish/Subscribe Communication in Delay Tolerant Networks," Proc. 10th ACM Symp. Modeling, Analysis, and Simulation of Wireless and Mobile Systems (MSWiM), pp. 225-234, 2007.

[37] Q. Yuan, I. Cardei, and J. Wu, "Predict and Relay: An Efficient Routing in Disruption-Tolerant Networks," Proc. ACM MobiHoc, pp. 95-104, 2009.

[38] J. Zhao, P. Zhang, G. Cao, and C. Das, "Cooperative Caching in Wireless P2P Networks: Design, Implementation, and Evaluation," IEEE Trans. Parallel \& Distributed Systems, vol. 21, no. 2, pp. 229-241, Feb. 2010.

[39] H. Zhu, L. Fu, G. Xue, Y. Zhu, M. Li, and L.M. Ni, "Recognizing Exponential Inter-Contact Time in VANETs," Proc. IEEE INFOCOM, 2010.

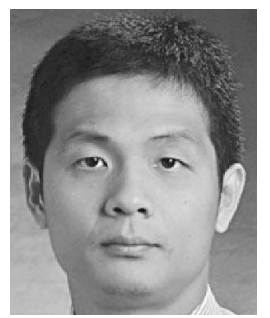

Wei Gao received the BE degree in electrical engineering from the University of Science and Technology of China in 2005 and the PhD degree in computer science from Pennsylvania State University in 2012. He is currently an assistant professor in the Department of Electrical Engineering and Computer Science at the University of Tennessee, Knoxville. His research interests include wireless and mobile network systems, mobile social networks, cyber-physical systems, and pervasive and mobile computing. $\mathrm{He}$ is a member of the IEEE.

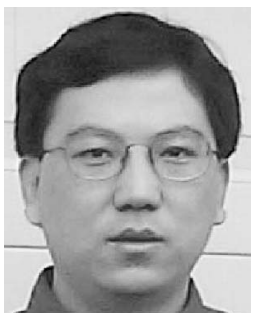

Guohong Cao received the BS degree in computer science from Xian Jiaotong University and the $\mathrm{PhD}$ degree in computer science from the Ohio State University in 1999. Since then, he has been with the Department of Computer Science and Engineering at Pennsylvania State University, where he is currently a professor. $\mathrm{He}$ has published more than 150 papers in the areas of wireless networks, wireless security, vehicular networks, wireless sensor networks, cache management, and distributed fault-tolerant computing. He has served on the editorial board of the IEEE Transactions on Mobile Computing, the IEEE Transactions on Wireless Communications, and the IEEE Transactions on Vehicular Technology, and has served on the organizing and technical program committees of many conferences, including TPC chair/cochair of IEEE SRDS 2009, MASS 2010, and INFOCOM 2013. He received the US National Science Foundation CAREER Award in 2001. He is a fellow of the IEEE.

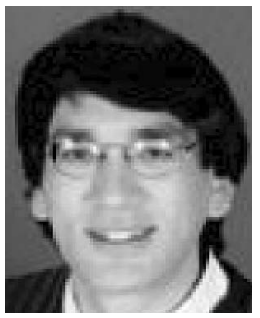

Arun lyengar received the $\mathrm{PhD}$ degree in computer science from the Massachusetts Institute of Technology (MIT). He does research and development in distributed computing, high availability, and web performance at IBM's T.J. Watson Research Center in Yorktown Heights, New York. His techniques for caching, load balancing, and serving dynamic content are widely used for web and distributed applications. $\mathrm{He}$ is the founding co-editor-in-chief of the $A C M$ Transactions on the Web, the chair of IFIP Working Group 6.4 on Internet Applications Engineering, and an IBM Master Inventor. He is a fellow of the IEEE.

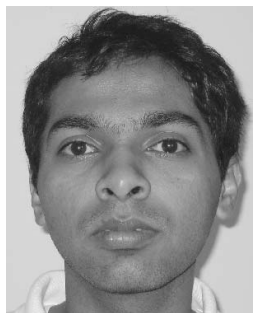

Mudhakar Srivatsa received the $\mathrm{PhD}$ degree in computer science from Georgia Tech. $\mathrm{He}$ is a research staff member in the Network Technologies Department at the Thomas J. Watson Research Center. His research interests primarily include network analytics and secure information flow. He serves as a technical area leader for secure hybrid networks research in the International Technology Alliance in Network and Information Sciences and as a principal investigator for information network research in the Network Science Collaborative Technology Alliance. He is a member of the IEEE.

For more information on this or any other computing topic, please visit our Digital Library at www.computer.org/publications/dlib. 